Objectives Determine the incidence and prevalence of nasal colonisation of swine veterinarians with S. aureus in the USA, and quantify associations between risks of $S$. aureus colonisation and infection and exposure to pigs.

Methods 1) Cross-sectional survey of swine veterinarians regarding general occupational health and safety. 2) Longitudinal bacteriological testing (nasal swabs) of 68 veterinarians sampled monthly for 18 months for S. aureus and MRSA carriage. Isolates are characterised of using spa typing (eGenomics and Ridom spa servers) and multilocus sequence typing. The veterinarians work in most major pig producing states of across the USA. Concurrent assessment of intensity of pig exposure, occurrence of skin and soft injuries, occurrence of $S$. aureus infections, and use of PPE is made monthly via survey.

Results Across the first 4 months of bacteriological testing, prevalence of both $S$. aureus (60-70\%) and MRSA (7-10\%) were above expected levels in the USA population $(30 \%, 3 \%$ respectively). Prevalence is significantly higher in veterinarians sampled within 48 hours of pig contact than after longer periods. Three spa types (539/t034 - ST398; 2/t_unknown - ST 5; 1435/t337 ST9 comprised $65 \%$ of all S. aureus isolates from swine veterinarians. The $3 \mathrm{spa} /$ sequence types have been reported as livestock associated' MRSA in Europe (ST398), Asia (ST9) and North America (ST5), but the majority of isolates from US veterinarians were methicillin sensitive (MSSA). Although minor skin injuries have been a frequent occurrence (5\% cumulative incidence monthly), clinical infections with MRSA or MSSA have not yet been reported.

Conclusions Swine veterinarians in the USA are frequently culture positive for $S$. aureus variants that are common in swine. Many exposure events appear to result in only transient colonisation. To date, risk of clinical S.aureus infection in this group appears to be modest, despite widespread exposure to the organisms and a high incidence of opportunity for infection via skin wounds.

\section{ANTIMICROBIAL RESISTANT BACTERIA IN PIG FARMERS IN THE NETHERLANDS - A STUDY ON ESBLS}

${ }^{1}$ W Dohmen, ${ }^{1}$ Bos, ${ }^{2}$ Cohen Stuart, ${ }^{3}$ Wagenaar, ${ }^{2}$ Bonten, ${ }^{1}$ Heederik. ${ }^{1}$ Institute for Risk Assessment Sciences, Utrecht University, Utrecht, Nederland; ${ }^{2}$ University Medical Center Utrecht, Utrecht, Nederland; ${ }^{3}$ Faculty of Veterinary Medicine, Utrecht University, Utrecht, Nederland

\subsection{6/oemed-2013-101717.346}

Objectives Farm animals may serve as a reservoir for (multi) resistant bacteria, such as extended-spectrum beta-lactamase (ESBL) producing Enterobacteriaceae. Animal to man transmission may occur through (in)direct contact during work, which may thus pose an occupational health hazard. In humans, infections with ESBL producing Enterobacteriaceae are associated with high mortality, morbidity and costs. We investigated the prevalence of carriage with ESBL producing Enterobacteriaceae in pig farmers, their family members, and their employees and associations between presence of ESBLs among animals and humans.

Methods Rectal swabs were taken from pigs on 40 Dutch conventional pig farms (60 per farm) and stool samples were obtained from 142 humans living and/or working on 34 of these farms (farmers, family members and employees). Sampling was repeated after 6 months. Presence of ESBL-producing bacteria was determined by selective plating and ESBL genes were analysed by microarray analysis and gene sequencing.
Questionnaires were used to determine antimicrobial use, hygiene, contact with animals and/or meat, and other relevant determinants.

Results ESBL genes, mostly CTX-M-1, TEM-52 en CTX-M-14, were determined in pig isolates on 17 farms $(43 \%)$ and in isolates from 8 participants (6\%). ESBL genes determined in farmers corresponded to those detected in pigs on their farm. ESBL carriage was more likely in farmers working on ESBL positive farms $(\mathrm{OR}>10)$. After 6 months ESBL genes were determined in isolates from 8 farmers (6\%). Only 2 of these farmers carried ESBL genes in both stool samples obtained with a 6 months interval.

Conclusions We found a strong association between ESBL carriage in farmers and ESBL occurrence on the farm. Repeated sampling indicates that ESBL carriage is not persistent in this human study population.

\section{DO CHANGES IN THE INCIDENCE OF OCCUPATIONALLY- ACQUIRED FOOD-BORNE ZOONOTIC INFECTIONS PRECEDE CHANGES IN THE WIDER POPULATION?}

${ }^{1} \mathrm{~S}$ J Stocks, ${ }^{2}$ Berk, ${ }^{1}$ McNamee, ${ }^{1}$ Carder, ${ }^{1}$ Agius. ' University of Manchester, Manchester, United Kingdom; ${ }^{2}$ University of Newcastle, Newcastle, United Kingdom

\subsection{6/oemed-2013-101717.347}

Objectives Recently the WHO estimated that up to $75 \%$ of emerging pathogens may be of zoonotic origin. Occupationally acquired zoonoses are of particular concern in food producing sectors because of the possibility of transmission through the food chain. We aim to investigate whether changes in incidence of occupationally-acquired zoonotic infections in agricultural and veterinary workers or food preparation and food retail workers are useful in predicting changes in the incidence of food-borne zoonotic infections in the wider population.

Methods In the UK infectious disease consultants report cases of occupationally acquired infectious disease to the Surveillance of Infectious Diseases at Work (SIDAW) scheme. Cases reported to SIDAW will be analysed using a 2 level negative binomial regression model adjusted for changes in the size of occupational group at risk as described elsewhere ${ }^{1}$. Following a target set by the UK Health Protection Agency (HPA) in 2001 to reduce the incidence of salmonella in UK produced chicken there has been a declining trend in outbreaks of food-borne salmonellosis. In contrast there has been little change in the number of outbreaks of food-borne camphylobacteriosis reported to the HPA over the same time. These trends reported to the HPA will be compared with SWORD data.

Results Between 1997 and 2010, 244 individual cases (209 outbreaks) of salmonellosis and 577 individual cases (522 outbreaks) of camphylobacterosis were reported to SWORD. Over the same time period 590 foodborne outbreaks of salmonellosis and 105 of camphylobacterosis were reported to the HPA. A simultaneous decline in the incidence of salmonellosis reported to SWORD and food-borne salmonellosis reported to the HPA was observed. These data will be analysed further to compare different occupational sectors.

Conclusion Reporting of occupational infectious diseases in some sectors may be a useful marker for changes in incidence occurring in the wider population.

\section{REFERENCE}

1 Turner et al. Occup Env Med 2012; 69:12 925-931 